\title{
Controllable nonlocal interactions between dark solitons in dipolar condensates
}

\author{
T. Bland, ${ }^{1}$ M. J. Edmonds, ${ }^{1}$ N. P. Proukakis, ${ }^{1}$ A. M. Martin, ${ }^{2}$ D. H. J. O'Dell, ${ }^{3}$ and N. G. Parker ${ }^{1}$ \\ ${ }^{1}$ Joint Quantum Centre Durham-Newcastle, School of Mathematics and Statistics, \\ Newcastle University, Newcastle upon Tyne, NE1 7RU, United Kingdom \\ ${ }^{2}$ School of Physics, University of Melbourne, Victoria 3010, Australia \\ ${ }^{3}$ Department of Physics and Astronomy, McMaster University, Hamilton, Ontario, L8S 4M1, Canada
}

(Received 2 September 2015; published 1 December 2015)

\begin{abstract}
We study the family of static and moving dark solitons in quasi-one-dimensional dipolar Bose-Einstein condensates, exploring their modified form and interactions. The density dip of the soliton acts as a giant antidipole which adds a nonlocal contribution to the conventional local soliton-soliton interaction. We map out the stability diagram as a function of the strength and polarization direction of the atomic dipoles, identifying both roton and phonon instabilities. Away from these instabilities, the solitons collide elastically. Varying the polarization direction relative to the condensate axis enables tuning of this nonlocal interaction between repulsive and attractive; the latter case supports unusual dark-soliton bound states. Remarkably, these bound states are themselves shown to behave like solitons, emerging unscathed from collisions with each other.
\end{abstract}

DOI: 10.1103/PhysRevA.92.063601

PACS number(s): 03.75.Lm, 05.45.Yv

\section{INTRODUCTION}

Solitons - waves which propagate without dispersion-are a paradigm of nonlinear physics, occurring across systems as varied as water, optical fibers, spin chains, the human circulatory system, and atomic Bose-Einstein condensates (BECs) [1]. Much of the soliton behaviors across these diverse systems, including their interactions and collisions, are universal [2]. The extreme controllability of the nonlinearity, dimensionality, and external potential in atomic BECs makes them an ideal playground for studying solitons [3-6], with experimental demonstrations of bright [7-12], dark [13-17], and dark-bright $[15,18]$ solitons. Moreover, these so-called matter-wave solitons provide insight into the interplay of solitons with quantum coherence [19] and have potential applications in interferometry [7,12,20,21], in surface force detection [22], and as robust quantum-information carriers [23].

In conventional media with local nonlinearity, soliton behavior is well established [1,24], e.g., solitons interact only at short distances (when their field profiles overlap). A current direction in soliton research is the addition of nonlocal nonlinearity. This enriches the soliton behavior, e.g., promoting nonlocal interactions between solitons, and offers prospects for studying wave analogs of particles with long-range interactions and emulating complex nonlinear networks [25]. To date, such nonlocal solitons have been observed in liquid crystals [26-28], thermononlinear optical fibers [25] and liquids [29], and optical ring fibers [30]. In these cases, the nonlocal response is provided by molecular reorientations, heat conduction, and acoustic waves, respectively. Typically these nonlinearities lead to retarded interactions.

The advent of BECs of atoms with sizable magnetic dipole moments- ${ }^{52} \mathrm{Cr}[31,32],{ }^{164} \mathrm{Dy}[33,34]$ and ${ }^{168} \mathrm{Er}$ [35] -allows for the study of superfluids with nonlocal nonlinearities [36], arising from the dipole-dipole (DD) atomic interactions. Remarkably, the ratio of local to nonlocal interactions can be directly tuned through Feshbach resonances [37]. This has opened the door to experimental observations of magnetostriction [38], anisotropic collapse [39], and selforganized droplet phases [34]. A host of predictions such as two-dimensional (2D) bright solitons [40] and stabilized threedimensional (3D) dark solitary waves [41] await experimental verification.

In this paper we obtain numerically the family of static and moving dark solitons in a quasi-one-dimensional (quasi-1D) dipolar BEC as stationary solutions in static and moving frames. We map out the stablity diagram and form of the soliton solutions as a function of the strength and polarization direction of the dipoles, covering experimentally relevant parameters. The dark solitons can acquire dramatically modified profiles, including ripples close to the roton instability. Moreoever we show analytically that the absence of atoms in a dark soliton causes it to act like a giant antidipole [42]. This induces a nonlocal contribution to the soliton-soliton interactions which scales as $1 / z^{3}$. This nonlocal contribution is effectively instantaneous and experimentally tunable in both strength and sign. When the nonlocal interaction between the solitons is attractive, the balance with the conventional repulsive soliton-soliton interaction supports bound states of two dark solitons, for which no analog exists in conventional condensates. We show that these bound states themselves have solitonlike properties in that they emerge unscathed from collisions with each other.

\section{THEORETICAL FRAMEWORK}

We consider a dilute, ultracold BEC of atoms with mass $m$ and polarized magnetic dipoles. At such low energies the interactions between the atoms can be described using a universal pseudopotential [36],

$$
U\left(\mathbf{r}-\mathbf{r}^{\prime}\right)=g \delta\left(\mathbf{r}-\mathbf{r}^{\prime}\right)+U_{\mathrm{dd}}\left(\mathbf{r}-\mathbf{r}^{\prime}\right) .
$$

The first term is a contact interaction accounting for the van der Waals interactions characterized by coefficient $g$. This gives rise to a local nonlinearity and mean-field potential $g n(\mathbf{r})$, where $n(\mathbf{r})$ is the atomic density. The second term gives the dipole-dipole interactions, with $U_{\mathrm{dd}}\left(\mathbf{r}-\mathbf{r}^{\prime}\right)=$ $C_{\mathrm{dd}}\left(1-3 \cos ^{2} \theta\right) / 4 \pi\left|\mathbf{r}-\mathbf{r}^{\prime}\right|^{3}$, where $\theta$ is the angle between the polarization direction and the interatom vector $\mathbf{r}-\mathbf{r}^{\prime}$, and $C_{\mathrm{dd}}$ (which is conventionally positive) characterizes the 
strength of the dipoles. This contribution to the interactions gives rise to a nonlocal nonlinearity and dipolar mean-field potential $\Phi(\mathbf{r})=\int U_{\mathrm{dd}}\left(\mathbf{r}-\mathbf{r}^{\prime}\right) n\left(\mathbf{r}^{\prime}\right) \mathrm{d} \mathbf{r}^{\prime}$. At the magic angle $\theta_{\mathrm{m}} \approx 53^{\circ}$ the dipole-dipole interactions disappear. For $\theta<\theta_{\mathrm{m}}$ the dipoles lie dominantly head to tail and attract, while for $\theta>\theta_{\mathrm{m}}$ they lie dominantly side by side and repel. However, by fast rotation of the polarization direction the time-averaged dipole-dipole interaction can be effectively reversed $\left(C_{\mathrm{dd}}<0\right)$, in which case the dipoles repel when head to tail and attract when side by side [43].

In three-dimensional geometries, dark solitons are prone to transverse excitation of the nodal line, the so-called snake instability. However, in quasi-one-dimensional geometries this instability is prevented and dark solitons become long lived $[5,17,44]$. We consider such a quasi-1D waveguide geometry, aligned along $z$. The confinement in the transverse directions is assumed to be harmonic with the form $V(\mathbf{r})=$ $m \omega_{\perp}^{2} r^{2} / 2$, where $\omega_{\perp}$ is the corresponding trap frequency. The quasi-1D limit is reached when this confinement is sufficiently strong $\left(\hbar \omega_{\perp} \gg \mu\right.$, where $\mu$ is the BEC chemical potential) that the condensate wave function approaches the harmonic oscillator state in the transverse direction $[45,46]$, with length scale $l_{\perp}=\sqrt{\hbar / m \omega_{\perp}}$. In this regime the $3 \mathrm{D}$ condensate can be parameterized via a $1 \mathrm{D}$ mean-field wave function $\psi(z, t)$ which obeys an effective 1D Gross-Pitaevskii equation (GPE) [47,48],

$$
i \hbar \partial_{t} \psi=\left(-\frac{\hbar^{2}}{2 m} \partial_{z z}^{2}+\frac{g}{2 \pi l_{\perp}^{2}}|\psi|^{2}+\Phi_{1 \mathrm{D}}\right) \psi .
$$

Here $\Phi_{1 \mathrm{D}}(z, t)=\int U_{1 \mathrm{D}}\left(z-z^{\prime}\right)|\psi|^{2} \mathrm{~d} z^{\prime}$ is the effective 1D dipolar potential, with associated pseudopotential [49],

$$
U_{1 \mathrm{D}}(u)=U_{0}\left[2 u-\sqrt{2 \pi}\left(1+u^{2}\right) e^{u^{2} / 2} \operatorname{erfc}\left(\frac{u}{\sqrt{2}}\right)+\frac{8}{3} \delta(u)\right],
$$

where $u=|z| / l_{\perp}$ and $U_{0}=C_{\mathrm{dd}}(1+3 \cos 2 \theta) / 32 \pi l_{\perp}^{3}$. While the above mean-field model ignores finite temperature effects, it is worth noting that the salient physical behavior of the (nondipolar) dark solitons observed experimentally to date are well described by the zero-temperature mean-field GrossPitaevskii model [13-17].

\section{STABILITY OF THE HOMOGENEOUS SYSTEM}

The stability of the homogeneous 1D dipolar BEC has been established in Refs. [47,48] for dipoles aligned along $z(\theta=0)$; here we map out the full parameter space $(0 \leqslant$ $\theta \leqslant \pi)$. The ground state has uniform $1 \mathrm{D}$ density $n_{0}$. Its chemical potential [the eigenvalue associated with the righthand side of Eq. (2)] is $\mu_{0}=n_{0} g / 2 \pi l_{\perp}^{2}+\Phi_{0}$, where the first term represents the van der Waals mean-field potential and $\Phi_{0}=-C_{\mathrm{dd}} n_{0}[1+3 \cos 2 \theta] / 24 \pi l_{\perp}^{2}$ represents the dipoledipole mean-field potential. The characteristic length and speed scales are the healing length $\xi=\hbar / \sqrt{m \mu_{0}}$ and speed of sound $c=\sqrt{\mu_{0} / m}$; a time scale follows as $\tau=\xi / c$. We parameterize the transverse BEC size via $\sigma=l_{\perp} / \xi$ where the quasi-1D approximation requires $\sigma \lesssim 1$ [45]. We take the arbitrary value $\sigma=0.2$ throughout this paper, but our qualitative findings are independent of $\sigma$.
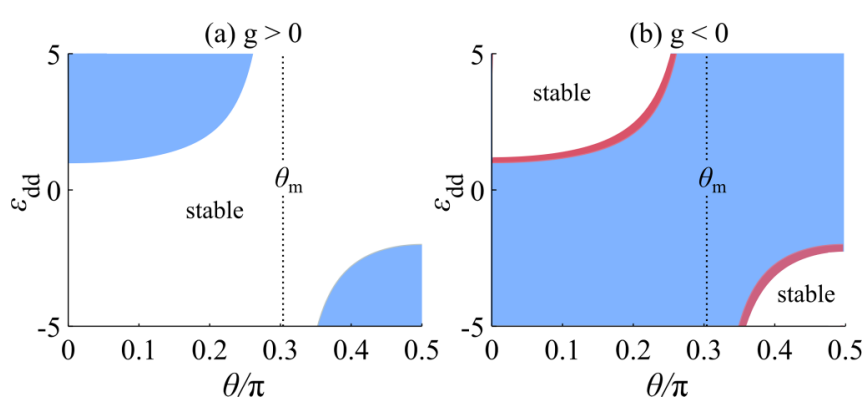

FIG. 1. (Color online) Stability diagrams in the $\left(\theta, \varepsilon_{\mathrm{dd}}\right)$ plane for the homogeneous quasi-1D dipolar BEC for (a) $g>0$ and (b) $g<0$. Depicted are the regions of stability (white), phonon instability (blue [dark gray]), and roton instability (red [light gray]). The magic angle $\theta_{\mathrm{m}}$ is highlighted.

It is convenient to parametrize the dipole strength via the ratio $\varepsilon_{\mathrm{dd}}=C_{\mathrm{dd}} / 3 g$ [36]. In the dipolar condensates produced to date, this ratio has the natural value $\varepsilon_{\mathrm{dd}}=0.16$ for ${ }^{52} \mathrm{Cr}$ [50], $\varepsilon_{\mathrm{dd}}=0.4$ for ${ }^{168} \operatorname{Er}[35]$, and $\varepsilon_{\mathrm{dd}}=1.4$ for ${ }^{164} \mathrm{Dy}[33,51]$. However, $g$ and $C_{\mathrm{dd}}$ can both be tuned in size and sign [36,43], and so we will typically consider a range of $\varepsilon_{\mathrm{dd}}$ with both negative and positive $g$ and $C_{\mathrm{dd}}$ (later, when considering soliton collisions and bound states, we will focus on the parameters for ${ }^{168} \mathrm{Er}$, and comment on the wider dependence on $\left.\varepsilon_{\mathrm{dd}}\right)$.

The homogeneous condensate is unstable for certain $C_{\mathrm{dd}}$, $g$ and $\theta[47,48]$; the stability in the $\left(\theta, \varepsilon_{\mathrm{dd}}\right)$ plane is depicted in Fig. 1(a) for $g>0$ and Fig. 1(b) for $g<0$. The condensate suffers two key instabilities: the phonon instability and the roton instability. The phonon instability refers to unstable growth of low $k$ modes. It arises when the net interactions become attractive, i.e., when $\mu_{0}<0$ (blue [gray] regions). Consider, for example, conventional dipoles $\left(C_{\mathrm{dd}}>0\right)$ : For $\theta=0$, the phonon instability arises when the attraction of the end-to-end dipoles dominates the van der Waals repulsion, while for $\theta=\pi / 2$ it arises when the repulsive side-byside dipoles are outweighed by attractive van der Waals interactions. The reverse is true for $C_{\mathrm{dd}}<0$. In both cases a long wavelength collapse is induced.

The dispersion relation can also feature a rotonlike dip at finite momenta $[47,48]$ which, for certain parameter regimes (red [light gray] regions in Fig. 1(b)) can touch zero energy, signaling the unstable growth of finite momentum modes, i.e., the roton instability. When deep in the $1 \mathrm{D}$ regime $(\sigma \ll 1$, as employed here) the roton instability arises for $g<0$ [48] [Fig. 1(b)]; however, for $\sigma \gtrsim 1$ the roton instability shifts to $g>0$, as predicted in Ref. [47].

\section{DARK-SOLITON SOLUTIONS}

Having established the stability of the homogeneous quasi1D dipolar BEC, we move on to the dark-soliton solutions themselves. It is known that the purely local GPE is integrable and supports a family of dark-soliton solutions for repulsive contact interactions $(g>0)[52,53]$,

$$
\psi_{\mathrm{s}}(z, t)=\sqrt{n_{0}}\left[\beta \tanh \frac{\beta(z-Z)}{\xi}+i \frac{v}{c}\right] e^{-i \mu t / \hbar}
$$


Here $\beta=\sqrt{1-v^{2} / c^{2}}$ and $Z(t)=z_{0}+v t$, where $z_{0}$ is the soliton's initial position and $v$ its speed. Stationary solitons have a node of zero density and a phase slip of $\pi$, whereas a $v=c$ soliton has no phase or density contrast from the background fluid. The soliton energy decreases with increasing speed [53], leading to the analog of a particle with negative effective mass [54]. Meanwhile, the density minimum of the soliton scales as $n_{\min } / n_{0}=\beta^{2}$ [5].

Noting that dark solitons are stationary solutions in a moving frame, we numerically seek solutions to the GPE (2) with a Galilean boost term $i \hbar v \partial_{z} \psi$ in the Hamiltonian. Discretizing $\psi$ on a $1 \mathrm{D}$ spatial grid (spacing $d z=0.1 \xi$ ) we minimize the discretized Hamiltonian with respect to changes in $\psi$ using a biconjugate method [55]. Starting with the nondipolar dark-soliton solution (4), this method leads to convergence to the required dipolar soliton solution, within the entire speed range $0 \leqslant v \leqslant c$. The numerical box (up to $\pm 800 \xi)$ is large enough to mimic the infinite limit.

The numerically obtained dark solutions propagate with constant speed and permanent form (no dispersion or radiative losses) when simulated within the laboratory-frame GPE [56], confirming their solitonic character. Dark solitons require the mean-field potential of the homogeneous system to be net repulsive [58,59], i.e., $\mu_{0}>0$, the same as the condition for phonon stability.

We illustrate the solutions through the $v=0$ soliton: Fig. 2 depicts its spatial density profile $n(z)$ as a function of $\varepsilon_{\mathrm{dd}}$ for the limiting angles (a) $\theta=0$ and (b) $\theta=\pi / 2$. The former is indicative of the general behavior for $\theta<\theta_{\mathrm{m}}$, and the latter for $\theta>\theta_{\mathrm{m}}$ (for $\theta=\theta_{\mathrm{m}}$ one recovers the nondipolar soliton throughout). The soliton size is characterized by the dipolar healing length $\xi$ (hence motivating this choice of units). Away from the unstable band of $\varepsilon_{\mathrm{dd}}$, the profile approximates the tanh-squared density profile of the nondipolar soliton. As the phonon instability is approached (for $g>0$ ) the profile diverges in width; this is related to a cancellation between the local interactions arising from the explicit van der Waals interactions and an implicit local contribution to the dipoledipole interactions [60]. Meanwhile, as the roton instability is approached, prominent density ripples form about the soliton core (see inset). These finite- $k$ corrugations arise due to the mixing of the roton with the soliton state, with analogous ripples arising for vortices [60-62]. With increasing speed, the soliton depth decreases in line with the nondipolar soliton, and the above qualitative behavior remains, albeit with weaker ripples as the speed is increased. The soliton phase profile is insensitive to $\varepsilon_{\mathrm{dd}}$ and $\theta$.

The soliton modifies the mean-field dipolar potential $\Phi_{1 \mathrm{D}}(z)$ in a nonlocal manner. We can glean some insight into this by considering the behavior at long range. Expanding the dipolar pseudopotential Eq. (3) around infinity gives $U_{1 \mathrm{D}}(u)=U_{0}\left(4|u|^{-3}-24|u|^{-5}+\cdots\right)$ [63]. Then, taking the nondipolar soliton (4) as an ansatz, the asymptotic form of $\Phi_{1 \mathrm{D}}$ follows as

$$
\Phi_{1 \mathrm{D}}(z) \simeq \Phi_{0}\left(1-\frac{\beta n_{0} \xi l_{\perp}^{3}}{e^{2 \beta z_{0} / \xi}\left|z_{0}-z\right|^{3}}\right)
$$

where $z_{0}$ is a short-range cutoff to account for the asymptotic behavior of the mean-field dipolar potential. The two terms represent the background and soliton contributions to $\Phi_{1 \mathrm{D}}$, respectively. The $1 / z^{3}$ decay of $\Phi_{1 \mathrm{D}}$ demonstrates that the soliton appears as a localized giant dipole, a key result of our work. This is intimately connected to the exponentially fast decay of the soliton density profile; at large distances the soliton profile scales as $n(z) \simeq\left(n_{0}-n_{\min }\right) e^{-z / \xi}$. In contrast, a slow power-law-decaying density profile would render the object as an extended dipole at all scales, as for vortices in 2D [60]. The negative sign on the soliton contribution suggests that the soliton may be viewed as a collection of antidipoles superposed on a homogeneous background of conventional dipoles. This observation will help us below to interpret the modified form of the soliton-soliton collisions as due to the interaction of these antidipoles.

\section{SOLITON-SOLITON COLLISIONS}

We can expect the nonlocality of the solitons to modify their interactions, which we examine next through their collisions. For simplicity we take the collisions to be symmetric, i.e., the same incoming speed for both solitons. The corresponding analytic two-soliton solution to the nondipolar 1D GPE is [5]

$$
\psi_{2 \mathrm{~s}}(z, t)=\{F(z, t) / G(z, t)\} \exp (-i \mu t / \hbar)
$$
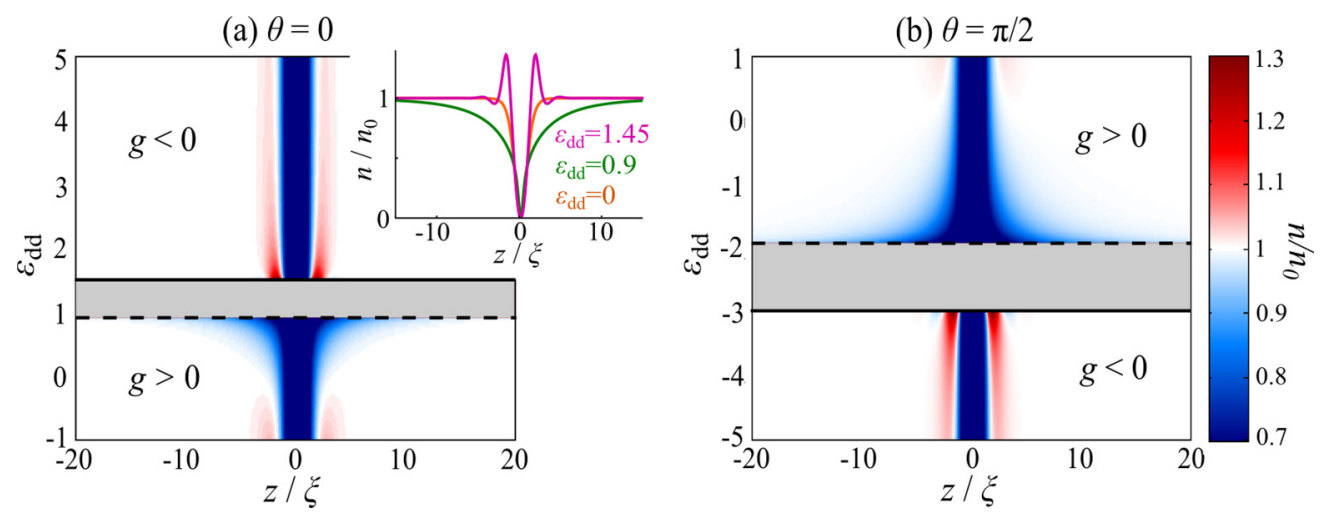

FIG. 2. (Color online) Density profiles $n(z)$ of the $v=0$ soliton for (a) $\theta=0$ and (b) $\theta=\pi / 2$, as a function of $\varepsilon_{\mathrm{dd}}$. The band (gray) of instability is bounded by the onset of the phonon and roton instabilities (dashed and solid lines, respectively). Inset: density profile for no dipoles $\left(\varepsilon_{\mathrm{dd}}=0\right)$ and close to the instabilities $\left(\varepsilon_{\mathrm{dd}}=0.9,1.45\right)$. Note that the unit of length $\xi$ is itself a function of $\varepsilon_{\mathrm{dd}}$ and $\theta$. 
(a) $\theta=0$

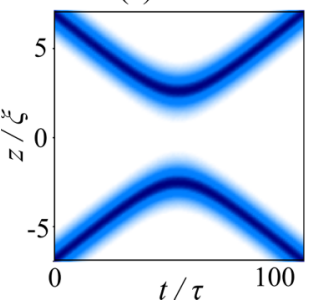

(b) $\theta=\theta_{\mathrm{m}}$

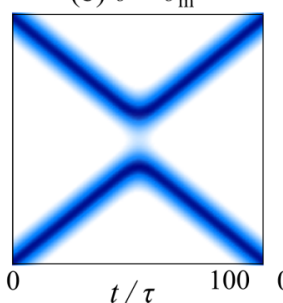

(c) $\theta=\pi / 2$

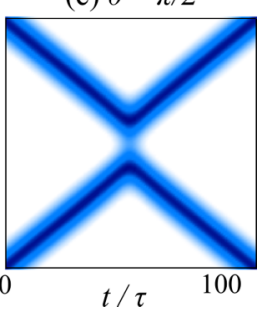

FIG. 3. (Color online) Collisions of dipolar dark solitons with incoming speeds $v=0.1 c$ for $\varepsilon_{\mathrm{dd}}=0.4$ and polarization angles (a) $\theta=0$, (b) $\theta=\theta_{\mathrm{m}}$, and (c) $\theta=\pi / 2$.

with

$$
\begin{aligned}
F(z, t)= & 2 \sqrt{n_{0}}\left[\left(1-2 v^{2} / c^{2}\right) \cosh (2 v \beta t / \xi)\right. \\
& -(v / c) \cosh (2 \beta z / \xi)+i 2(v / c) \beta \sinh (2 v \beta t / \xi)], \\
G(z, t)= & 2 \cosh (2 v \beta t / \xi)+2(v / c) \cosh (2 \beta z / \xi) .
\end{aligned}
$$

Conventionally, solitons are known to repel during their collision with two regimes of dynamics: For low incoming speeds $v<0.5 c$ the solitons reflect at short range, with a sudden large repulsion akin to hard-sphere collisions, while for higher speeds $v \geqslant 0.5 c$ the solitons transmit $[17,64]$. We focus on slow collisions $v=0.1 c$, commenting on the wider speed dependence below. We adopt fixed dipole parameters corresponding to ${ }^{168} \operatorname{Er}[35]$, i.e., $\varepsilon_{\mathrm{dd}}=0.4$. Figure 3 shows some example soliton collisions, simulated via the 1D dipolar GPE [56], for different values of polarization angle $\theta$. The solitons emerge from the collisions with unchanged profile and no radiative loss, further supporting their solitonic character. In Fig. 3(b), the magic angle $\theta_{\mathrm{m}}$, one recovers the nondipolar dynamics in which the solitons bounce at short range [64]. In Fig. 3(a), $\theta<\theta_{\mathrm{m}}$, the soliton repulsion is significantly enhanced, turning them at increased separation, indicative of the role of the nonlocal interactions. Here the dipoles are net attractive but the solitons themselves (which, recall, behave as antidipoles) are effectively net repulsive. Vice versa, in Fig. 3(c), $\theta>\theta_{\mathrm{m}}$, the dipole-dipole interactions introduce an attraction between solitons; however, the effect of this is barely visible due to the dominance of the hard-sphere-like repulsion at short range.

The nonlocal repulsive and attractive modifications to the soliton collisions become more pronounced with increasing $\varepsilon_{\mathrm{dd}}$ and $\sigma$ due to the increasing strength of the effective 1D dipole-dipole interactions. Meanwhile, they become less pronounced with increasing soliton speeds; this is due to the reduced mean-field potential generated by faster and shallower solitons, as evident from the $\beta$ dependence in Eq. (5). While the elastic collisions in Fig. 3 are representative of most of the stable $\left(\theta, \varepsilon_{\mathrm{dd}}\right)$ space, collisions become inelastic close to the phonon instability or roton instability; then sound waves are shed during the collision, causing a slight (few percent) increase in their outgoing speeds.

\section{TWO-SOLITON INTERACTION POTENTIAL AND BOUND STATES}

We can further understand the soliton-soliton interaction through an effective particlelike interaction potential. We calculate the soliton's energy, relative to the background, as

$$
E_{\mathrm{tot}}=E_{0}+E_{\mathrm{dd}},
$$

where

$$
E_{0}=\int\left[\frac{\hbar^{2}}{2 m}\left|\partial_{z} \psi\right|^{2}+\frac{g}{2}\left(|\psi|^{2}-n_{0}\right)^{2}\right] d z,
$$

is the nondipolar energy (sum of kinetic and van der Waals interaction energies) and

$$
E_{\mathrm{dd}}=\int \frac{1}{2} \Phi_{1 \mathrm{D}}|\psi|^{2} d z
$$

is the dipolar contribution. For two solitons at $z_{1}$ and $z_{2}$ (defined by their density minima), with separation $q=\mid z_{1}-$ $z_{2} \mid$, we define the interaction energy as

$$
V(q)=E_{\mathrm{tot}}\left(z_{1}, z_{2}\right)-E_{\mathrm{tot}}\left(z_{1}\right)-E_{\mathrm{tot}}\left(z_{2}\right) .
$$

We estimate $V(q)$ semianalytically based on the nondipolar two-soliton solution with small incoming speed (for larger speeds, the effective masses of the solitons change considerably during the collision, complicating this particlelike picture). Figure 4(a) shows the soliton-soliton interaction potential, again for ${ }^{168} \mathrm{Er}$ parameters. The nondipolar contribution $V_{0}$ (dashed [red] line) dominates only at short range, consistent with the repulsive bouncing of two nondipolar solitons [Fig. 3(b)]. For $\theta=0$, the dipolar interaction potential $V_{\text {dd }}$ (black dot-dashed line) is repulsive and nonlocal, consistent with the bouncing at increasing separation observed in Fig. 3(a). However, for $\theta=\pi / 2, V_{\mathrm{dd}}$ (dotted [blue] line) is attractive at long range. The playoff between this nonlocal attraction and the short-range repulsion from $V_{0}$ conspires
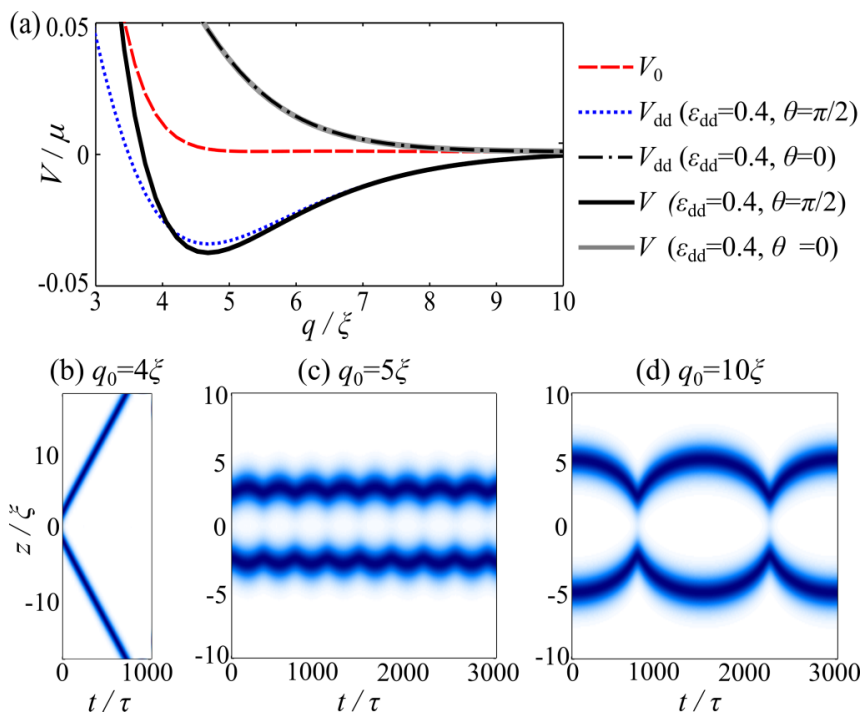

FIG. 4. (Color online) (a) Soliton-soliton interaction potential $V$ as a function of separation $q$ for $\varepsilon_{\mathrm{dd}}=0.4$ and $\theta=\pi / 2$ (estimated using the nondipolar two-soliton solution in the low speed limit with arbitrary value $v=0.001 c$ ). The local minimum indicates a bound state. The nondipolar $V_{0}$ and dipolar $V_{\mathrm{dd}}$ contributions are indicated. (b)-(d) Corresponding GPE dynamics starting from two stationary solitons a distance $q_{0}$ apart. For (a) $q_{0}=4 \xi$, the solitons repel, while for (b) $q_{0}=5 \xi$ and (c) $q_{0}=10 \xi$, bound state oscillations are evident. 
to form a total potential (solid black line) analogous to the Lennard-Jones interatomic potential: an energy minimum at finite $q$, with rapidly increasing potential at short range and slowly increasing potential at larger range. This raises the prospect of forming a two-soliton bound state, analogous to a diatomic molecule.

The possibility of supporting a two-soliton bound state is probed through GPE simulations starting with two stationary solitons a distance $q_{0}$ apart, shown in Figs. 4(b)-4(d). For sufficiently small $q_{0}(\mathrm{a})$, the solitons initially feel a strongly repulsive short-range interaction, repel, and acquire sufficient kinetic energy to escape to infinity. For greater separations, e.g., Figs. 4(b) and 4(c), the solitons initially have negative potential energy and are restricted to execute oscillations in $q$ about the potential minimum, analogous to vibrational modes of a diatomic molecule. For large initial separations $q_{0} \gg q_{\mathrm{min}}$, the solitons experience a weak gradient in $V(q)$ and undergo slow oscillations.

Well away from the roton instability and phonon instability, these bound-state oscillations persist ad infinitum (within the zero-temperature GPE). However, close to these instabilities, repeated sound emission during the collisions leads to the counterintuitive situation where the solitons increase their oscillation amplitude and ultimately escape the bound state by losing energy; this is related to the negative effective mass of the soliton and is analogous to antidamping effects in external potentials [54].

We further investigate the properties of the bound state by considering its interaction with another bound state. Figure 5 depicts the collisions of two counterpropagating bound states at low [Fig. 5(a)] and high [Fig. 5(b)] speed. These moving bound states are formed by repeating the above method for forming bound states, but where both initial solitons have the same nonzero speed. First, it is worth observing from the plot that the bound states are stable to center-of-mass motion at constant speed. For both low and high speeds, the two bound states emerge unscathed from the collision, with their original speeds and with no radiative losses. The only net difference is that the outgoing bound states both feature a translational offset, termed a phase shift. This is considerably larger for the low-speed case and is just visible in the high-speed collision. At low speed, the bound states appear to bounce off each other, just like in the collision of two slow dark solitons [Fig. 3], while
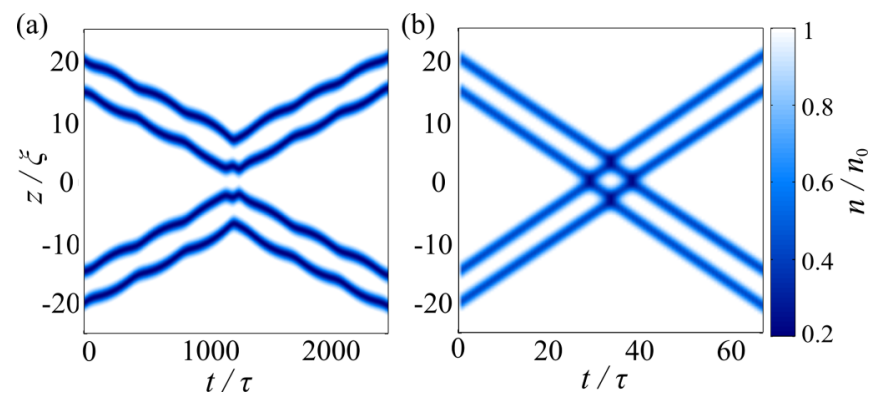

FIG. 5. (Color online) Collision of two counterpropagating bound states, with speeds (a) $v=0.01 c$ and (b) $v=0.5 c$ In both cases, the bound states emerge unscathed from the collision, with the only net effect being a phase shift of the outgoing waves (for the high-speed case this phase shift is only just visible). at high speed the bound states appear to pass through each other, again analogous to the corresponding behavior of two fast colliding dark solitons [5]. Note that for the high-speed case, the solitons in each bound state appear to move in parallel; this is simply because the period of the bound-state oscillation is considerably longer than the time scale of the figure and the collision. We have repeated these simulations over a wider rage of incoming speeds and find the same qualitative solitonlike behavior throughout. These results demonstrate the striking property that the bound states themselves behave like solitons in their interactions with other bound states.

Furthermore, analyzing the collision between a bound state and a single incident dark soliton shows the same behavior, with the single soliton and bound state emerging unscathed from the collision, barring a phase shift.

\section{CONCLUSIONS}

In summary, we have studied self-trapped nonlocal dark solitons supported within quasi-1D dipolar BECs. The solitons acquire modified profiles, including ripples associated with roton excitations. The solitons approximate giant localized dipoles and have nonlocal soliton-soliton interactions, controllable through the direction of polarization of the dipoles. When attractive, and in combination with the conventional short-range repulsive interaction, unconventional dark-soliton bound states can be realized. These bound states are stable to center-of-mass propagation at constant speed. Remarkably, they act themselves like solitons during collisions, emerging with unchanged form and speed.

Some of these effects are analogous to predictions for vortices in two-dimensional dipolar condensates [60]. There, vortices bear similar ripples about the core, with these ripples being a common manifestation in dipolar condensates in the vicinity of a sharply varying density profile. However, the different density profiles of solitons and vortices lead to signficantly different results. For a vortex, the profile scales asymptotically as $1 / r^{2}$ back to the background density; this is sufficiently slowly varying that it renders the vortex as an extended dipole at all scales. In contrast, the asymptotic part of the soliton density profile decays exponentially fast to the background, such that the soliton resembles a localized dipole at long range. These differences, in turn, lead to different functional forms of their interaction potentials: For solitons the interaction potential scales with the dipolar form $1 / r^{3}$ at long range, while for vortices the most slowly decaying terms go as $1 / r^{2}$ and $\ln (r) / r^{3}$. For vortices this is in addition to the usual long-range, isotropic interaction arising from hydrodynamic effects.

The above soliton behavior occurs robustly across a wide parameter space, accessible to dipolar BEC experiments [3133,35] and with current dark-soliton generation and imaging capabilities [15-17]. These dipolar dark solitons extend the range of physics of dark solitons as mesoscopic probes of quantum physics [19] to include the interplay with magnetism. Moreover, the effectively instantaneous nonlocal soliton interaction and its capacity to be experimentally tuned at will offer intriguing possibilities for the controlled study of nonlocality in complex networks [25], soliton gases [65], and supersolitons [66]. 
Note Added. Recently, we became aware of Ref. [67], which obtains results related to ours.

Data supporting this publication is openly available under an Open Data Commons Open Database License [68].

\section{ACKNOWLEDGMENTS}

This work was supported by the Engineering and Physical Sciences Research Council (Grant No. EP/M005127/1). D.O. also acknowledges support from the Natural Sciences and Engineering Research Council (NSERC) of Canada.
[1] T. Dauxois and M. Peyrard, Physics of Solitons (Cambridge University Press, Cambridge, UK, 2006).

[2] G. I. Stegeman and M. Segev, Science 286, 1518 (1999).

[3] P. G. Kevrekidis, D. J. Frantzeskakis, and R. Carretero-Gonzalez (eds.), Emergent Nonlinear Phenomena in Bose-Einstein Condensates (Springer, Berlin, 2008).

[4] F. Kh. Abdullaev, A. Gammal, A. M. Kamchatnov, and L. Tomio, Int. J. Mod. Phys. B 19, 3415 (2005).

[5] D. J. Frantzeskakis, J. Phys. A 43, 213001 (2010).

[6] T. P. Billam, A. L. Marchant, S. L. Cornish, S. A. Gardiner, and N. G. Parker, in Spontaneous Symmetry Breaking, SelfTrapping, and Josephson Oscillations, Progress in Optical Science and Photonics Vol. 1, edited by B. A. Malomed (Springer, Heidelberg, 2013), p. 403.

[7] K. E. Strecker et al., Nature (London) 417, 150 (2002).

[8] L. Khaykovich et al., Science 296, 1290 (2002).

[9] B. Eiermann, Th. Anker, M. Albiez, M. Taglieber, P. Treutlein, K.-P. Marzlin, and M. K. Oberthaler, Phys. Rev. Lett. 92, 230401 (2004).

[10] S. L. Cornish, S. T. Thompson, and C. E. Wieman, Phys. Rev. Lett. 96, 170401 (2006).

[11] A. L. Marchant, T. P. Billam, T. P. Wiles, M. M. H. Yu, S. A. Gardiner, and S. L. Cornish, Nat. Commun. 4, 1865 (2013).

[12] G. D. McDonald, C. C. N. Kuhn, K. S. Hardman, S. Bennetts, P. J. Everitt, P. A. Altin, J. E. Debs, J. D. Close, and N. P. Robins, Phys. Rev. Lett. 113, 013002 (2014).

[13] S. Burger, K. Bongs, S. Dettmer, W. Ertmer, K. Sengstock, A. Sanpera, G. V. Shlyapnikov, and M. Lewenstein, Phys. Rev. Lett. 83, 5198 (1999).

[14] J. Denschlag et al., Science 287, 97 (2000).

[15] C. Becker et al., Nat. Phys. 4, 496 (2008).

[16] S. Stellmer, C. Becker, P. Soltan-Panahi, E. M. Richter, S. Dorscher, M. Baumert, J. Kronjager, K. Bongs, and K. Sengstock, Phys. Rev. Lett. 101, 120406 (2008).

[17] A. Weller, J. P. Ronzheimer, C. Gross, J. Esteve, M. K. Oberthaler, D. J. Frantzeskakis, G. Theocharis, and P. G. Kevrekidis, Phys. Rev. Lett. 101, 130401 (2008).

[18] C. Hamner, J. J. Chang, P. Engels, and M. A. Hoefer, Phys. Rev. Lett. 106, 065302 (2011).

[19] J. Anglin, Nat. Phys. 4, 437 (2008).

[20] A. Negretti and C. Henkel, J. Phys. B 37, L385 (2004).

[21] J. L. Helm, S. L. Cornish, and S. A. Gardiner, Phys. Rev. Lett. 114, 134101 (2015).

[22] S. L. Cornish et al., Phys. D (Amsterdam, Neth.) 238, 1299 (2009).

[23] M. Lewenstein and B. A. Malomed, New. J. Phys. 11, 113014 (2009).

[24] P. G. Drazin and R. S. Johnson, Solitons: An Introduction (Cambridge University Press, Cambridge, UK, 1989).

[25] C. Rotschild, B. Alfassi, O. Cohen, and M. Segev, Nat. Phys. 2, 769 (2006).
[26] M. Peccianti, K. A. Brzdkiewicz, and G. Assanto, Opt. Lett. 27, 1460 (2002).

[27] L. G. Cao, Y. J. Zheng, W. Hu, P. B. Yang, and Q. Guo, Chin. Phys. Lett. 26, 064209 (2009).

[28] A. Piccardi, A. Alberucci, N. Tabiryan, and G. Assanto, Opt. Lett. 36, 1356 (2011).

[29] A. Dreischuh, D. Neshev, D. E. Petersen, O. Band, and W. Krolikowski, Phys. Rev. Lett. 96, 043901 (2006).

[30] J. K. Jang, M. Erkintalo, S. G. Murdoch, and S. Coen, Nat. Photon. 7, 657 (2013).

[31] A. Griesmaier, J. Werner, S. Hensler, J. Stuhler, and T. Pfau, Phys. Rev. Lett. 94, 160401 (2005).

[32] Q. Beaufils, R. Chicireanu, T. Zanon, B. Laburthe-Tolra, E. Maréchal, L. Vernac, J. C. Keller, and O. Gorceix, Phys. Rev. A 77, 061601(R) (2008).

[33] M. Lu, N. Q. Burdick, S. H. Youn, and B. L. Lev, Phys. Rev. Lett. 107, 190401 (2011).

[34] H. Kadau, M. Schmitt, M. Wenzel, C. Wink, T. Maier, I. FerrierBarbut, and T. Pfau, arXiv:1508.05007.

[35] K. Aikawa, A. Frisch, M. Mark, S. Baier, A. Rietzler, R. Grimm, and F. Ferlaino, Phys. Rev. Lett. 108, 210401 (2012).

[36] T. Lahaye, C. Menotti, L. Santos, M. Lewenstein, and T. Pfau, Rep. Prog. Phys. 72, 126401 (2009).

[37] T. Koch, T. Lahaye, J. Metz, B. Frölich, A. Griesmaier, and T. Pfau, Nat. Phys. 4, 218 (2008).

[38] J. Stuhler, A. Griesmaier, T. Koch, M. Fattori, T. Pfau, S. Giovanazzi, P. Pedri, and L. Santos, Phys. Rev. Lett. 95, 150406 (2005).

[39] T. Lahaye, J. Metz, B. Frohlich, T. Koch, M. Meister, A. Griesmaier, T. Pfau, H. Saito, Y. Kawaguchi, and M. Ueda, Phys. Rev. Lett. 101, 080401 (2008).

[40] P. Pedri and L. Santos, Phys. Rev. Lett. 95, 200404 (2005).

[41] R. Nath, P. Pedri, and L. Santos, Phys. Rev. Lett. 101, 210402 (2008).

[42] M. Klawunn, R. Nath, P. Pedri, and L. Santos, Phys. Rev. Lett. 100, 240403 (2008)

[43] S. Giovanazzi, A. Gorlitz, and T. Pfau, Phys. Rev. Lett. 89, 130401 (2002).

[44] A. Muryshev, G. V. Shlyapnikov, W. Ertmer, K. Sengstock, and M. Lewenstein, Phys. Rev. Lett. 89, 110401 (2002).

[45] A. Görlitz, J. M. Vogels, A. E. Leanhardt, C. Raman, T. L. Gustavson, J. R. Abo-Shaeer, A. P. Chikkatur, S. Gupta, S. Inouye, T. Rosenband, and W. Ketterle, Phys. Rev. Lett. 87, 130402 (2001).

[46] N. G. Parker and D. H. J. O’Dell, Phys. Rev. A 78, 041601(R) (2008)

[47] S. Giovanazzi and D. H. J. O’Dell, Eur. Phys. J. D 31, 439 (2004).

[48] S. Sinha and L. Santos, Phys. Rev. Lett. 99, 140406 (2007).

[49] F. Deuretzbacher, J. C. Cremon, and S. M. Reimann, Phys. Rev. A 81, 063616 (2010); 87, 039903(E) (2013). 
[50] A. Griesmaier, J. Stuhler, T. Koch, M. Fattori, T. Pfau, and S. Giovanazzi, Phys. Rev. Lett. 97, 250402 (2006).

[51] Y. Tang, A. Sykes, N. Q. Burdick, J. L. Bohn, and B. L. Lev, Phys. Rev. A 92, 022703 (2015).

[52] V. E. Zakharov and A. B. Shabat, Sov. Phys. JETP 37, 823 (1973).

[53] Y. S. Kivshar and B. Luther-Davies, Phys. Rep. 298, 81 (1998).

[54] Th. Busch and J. R. Anglin, Phys. Rev. Lett. 84, 2298 (2000).

[55] T. Winiecki, Ph.D. thesis, Durham University, 2001 (unpublished).

[56] We numerically propagate the GPE in time using a CrankNicolson finite-difference scheme [57].

[57] X. Antoine, W. Bao, and C. Besse, Comp. Phys. Commun. 184, 2621 (2013).

[58] J. Cuevas, B. A. Malomed, P. G. Kevrekidis, and D. J. Frantzeskakis, Phys. Rev. A 79, 053608 (2009).

[59] P. A. Andreev and L. S. Kuz'menkov, Eur. Phys. J. D 68, 270 (2014).
[60] B. C. Mulkerin, R. M. W van Bijnen, D. H. J. ODell, A. M. Martin, and N. G. Parker, Phys. Rev. Lett. 111, 170402 (2013); B. C. Mulkerin, D. H. J. O’Dell, A. M. Martin, and N. G. Parker, J. Phys.: Conf. Ser. 497, 012025 (2014).

[61] S. Yi and L. You, Phys. Rev. A 63, 053607 (2001).

[62] R. M. Wilson, S. Ronen, J. L. Bohn, and H. Pu, Phys. Rev. Lett. 100, 245302 (2008).

[63] M. Abramowitz and I. A. Stegun, Pocketbook of Mathematical Functions (Verlag Harri Deutsch, Frankfurt, 1984).

[64] G. Theocharis, A. Weller, J. P. Ronzheimer, C. Gross, M. K. Oberthaler, P. G. Kevrekidis, and D. J. Frantzeskakis, Phys. Rev. A 81, 063604 (2010).

[65] G. A. El and A. M. Kamchatnov, Phys. Rev. Lett. 95, 204101 (2005).

[66] D. Novoa, B. A. Malomed, H. Michinel, and V. M. Perez-Garcia, Phys. Rev. Lett. 101, 144101 (2008).

[67] K. Pawłowski and K. Rzążewski, New J. Phys. 17, 105006 (2015).

[68] Newcastle University Data, http://doi.org/10.17634/137139-1. 


\section{University Library}

\section{- M M N E R VA A gateway to Melbourne's research publications}

Minerva Access is the Institutional Repository of The University of Melbourne

Author/s:

Bland, T;Edmonds, MJ;Proukakis, NP;Martin, AM;O'Dell, DHJ;Parker, NG

Title:

Controllable nonlocal interactions between dark solitons in dipolar condensates

Date:

2015-12-01

Citation:

Bland, T., Edmonds, M. J., Proukakis, N. P., Martin, A. M., O'Dell, D. H. J. \& Parker, N. G. (2015). Controllable nonlocal interactions between dark solitons in dipolar condensates. PHYSICAL REVIEW A, 92 (6), https://doi.org/10.1103/PhysRevA.92.063601.

Persistent Link:

http://hdl.handle.net/11343/58853 